Document downloaded from:

http://hdl.handle.net/10251/117076

This paper must be cited as:

Aragón Revuelta, P.; Noguera Murray, PS.; Bañuls Polo, M.; Puchades, R.; Maquieira Catala, A.; González Martínez, MÁ. (2018). Modulating receptor-ligand binding in biorecognition by setting

surface wettability. Analytical and Bioanalytical Chemistry. 410(23):5723-5730. https://doi.org/10.1007/s00216-018-1247-8

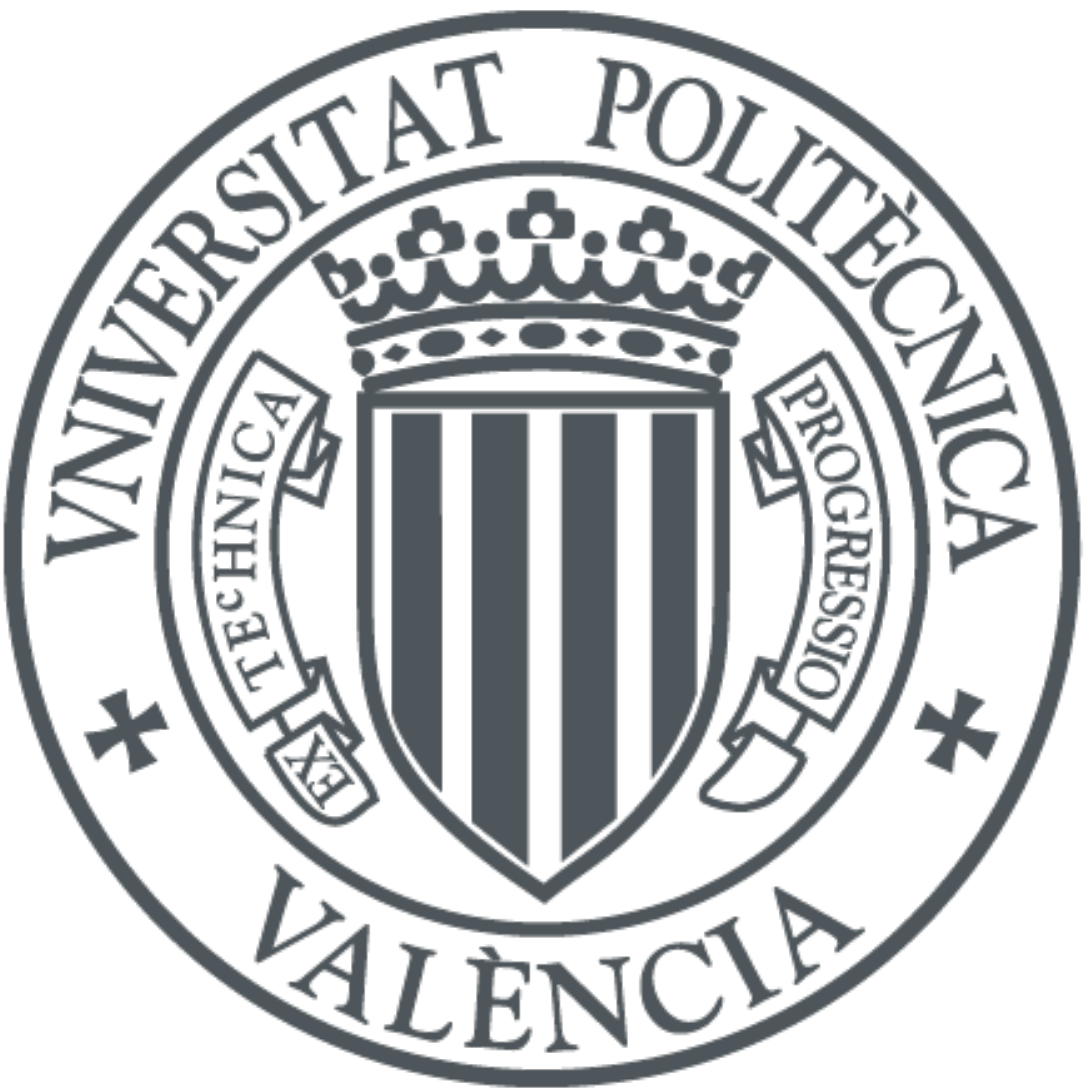

The final publication is available at

http://doi.org/10.1007/s00216-018-1247-8

Copyright Springer-Verlag

Additional Information 


\title{
Modulating receptor-ligand binding in biorecognition by setting surface wettability
}

\author{
Pilar Aragón, Patricia Noguera, María-José Bañuls, Rosa Puchades, Ángel \\ Maquieira*, Miguel Ángel González-Martínez*
}

Instituto Interuniversitario de Investigación de Reconocimiento Molecular y Desarrollo Tecnológico (IDM), Universitat Politècnica de València, Universitat de València.

Departamento de Química, Universitat Politècnica de Valencia, Camino de Vera s/n, 46022 Valencia, Spain

Ángel Maquieira: amaquieira@qim.upv.es. Phone +34 963877007

Miguel Ángel González-Martínez: mgonzal1@qim.upv.es. Phone +34 963877007

\begin{abstract}
Modulation of support wettability used for microarray format biosensing has led to an improvement of results. Hydrophobicity of glass chips was set by derivatizing with single vinyl organosilanes of different chain length and silane mixtures. Thiol-ene photochemical linking has been used as effective chemistry for covalent anchoring of thiolated probes. Lowest unspecific binding and highest signal intensity and SNR were obtained with large hydrocarbon chain $\left(\mathrm{C}_{22}\right)$ silanes or a shorter one $\left(\mathrm{C}_{10}\right)$ containing fluorine atoms. SNR resulting values are improved, reaching levels higher than 1500 in some cases, when using vinyl silanes modified with $1 \% \mathrm{C}_{10}$ alkyl fluorinated one, because mild hydrophobicity was achieved (water contact angle ca. $110^{\circ}$ ) for all silanes, including the short $\mathrm{C}_{2}$ and $\mathrm{C}_{3}$, thus giving rise to smaller and better defined array spots. In addition unspecific binding of reagents and targets was totally withdrawn. Hence, good-performing surfaces for biosensing applications can be built using appropriate organosilane reagent selection, including fluorinated ones.
\end{abstract}

KEYWORDS Surface wettability, organosilane, probe anchoring, thiol-ene click reaction, biosensing.

ORCID codes:

Pilar Aragon: 0000-0001-7815-9562

Patricia Noguera: 0000-0001-7155-0710

María-José Bañuls: 0000-0002-2422-7731

Rosa Puchades: 0000-0002-9329-1593

Ángel Maquieira: 0000-0003-4641-4957

Miguel Ángel González-Martínez: 0000-0002-5104-1262 


\section{Introduction}

Biosensing on solid substrates in microarray formats are popular, due to the high throughput screening achieved, so studies leading to understand and improve the performances of biomolecular recognition events on surfaces are always welcome, their main objective being maximizing the ligand binding to the receptor and minimizing non desired interactions of ligand or interfering species. Clearly, the choice of an adequate support and immobilization method can decide the biosensor analytical performances, and its applicability [1].

The study of surface properties for biosensing can be addressed on the wettability, that is the tendency in terms of adhesive and cohesive forces for keeping physical contact between surface and water, establishing an equilibrium solid-liquid-air. The surface wettability is established by measuring the contact angle of a liquid droplet on the flat horizontal surface (usually water contact angle WCA), and can affect the different reacting species to be on the areas where binding events take place (or not). It is therefore interesting to modulate the wettability of the surfaces involved in heterogeneous biorecognition events [2], since water is the natural solvent of these assays.

In fact, the relation between binding assay performances and surface properties is of great importance mainly because non-specific binding, an integral aspect of the performance of all kinds of bioassays, depends on support features [3]. In a recent study about the effect of hydrophobicity in DNA microarrays [4], different hydrophobicity degree surfaces were assayed, concluding that mild hydrophobicity is optimal for that application, and that the study of wettability influence should be carried out with a good ratio of hydrophobicity/hydrophilicity but avoiding extremes.

Wettability of a biosensing surface depends mainly on the nature of the raw material and the functionalization applied. The support requires physical and chemical stability, compatibility with the transduction system, and capability of anchoring the bioreceptor [5]. Glass is a common and representative support, cheap, available in different textures, and it can be derivatized via well-known organosilane chemistry for covalent attachment of probes [6].

The goal of this work is setting glass wettability by selecting and combining silanes, determining the surface free energy values, and studying their influence on the binding performances. To achieve this aim, biotin-streptavidin biorecognition was first selected as a proof of concept model due to its high affinity and selectivity. Surface was biotinylated by means of the thiol-ene click reaction [7] and Cy5-labelled protein streptavidin was added in solution. For modulating wettability, vinyl-ended silanes having hydrocarbon chains of different length were assayed, as well as fluorinated derivatives to enhance hydrophobicity due to the presence of surface fluorine atoms [8], and mixtures of both. Finally, in order to emphasize the relevance of this study, a more practical biosensing system (indirect immunoassay format) was assayed, consisting of the binding of anti-biotin antibody to biotinylated surface, followed by the recognition of a labelled secondary antibody. 


\section{Materials and methods}

\section{Materials and reagents}

Microscope slides (Premium Line, Labbox, Mataró, Spain) were cut to achieve smooth and frosted glass chips. Vinyl hydrocarbon alcoxy silanes with different chain length, purchased from Gelest (Morrisville, PA) and Acros (Geel, Belgium), were vinyltrimethoxysilane ( $\mathrm{C}_{02}$-VTMS), allyltrimethoxysilane (C03-ATMS), 3-butenyltriethoxysilane (C04-BTES), 7-octenyltrimethoxysilane (C $\mathrm{C}_{08}$-OTMS), 10-undecenyltrimethoxysilane ( $\mathrm{C}_{11}$-UTMS), and docosenyltriethoxysilane ( $\mathrm{C}_{22}$-DTES). Other quasi-fluorinated silanes used were dodecafluorodec-9-ene-1-yltrimethoxysilane (C $10 \mathrm{~F}-\mathrm{DFMS})$ and 1H,1H,2H,2H-perfluorodecyltriethoxysilane (C10F-FTES, not finished in vinyl).

Buffers used were PBS (10 mM sodium phosphate, $137 \mathrm{mM}$ sodium chloride, $2.7 \mathrm{mM}$ potassium chloride, pH 7.4), PBSG (PBS with 5\% glycerol) and PBST (PBS with 0.05\% Tween 20). Two biotin derivatives described in Electronic Supplementary Material (ESM, S1) were synthesized according to the procedure described by Weinrich et al. [9], one of them (thiolated biotin, TB) derivatized with thiol and the other one (control biotin CB) only with a spacer arm. Cy5-streptavidin conjugate was from GE Healthcare (Amersham, Buckinghamshire, UK). Rabbit polyclonal anti-biotin antibody was from Abcam (Cambridge, UK) and Alexa-Fluor 647-labelled goat anti-rabbit IgG was from Life Technologies (Eugene, OR).

\section{Apparatus}

Drop microarrays were laid on the chip surface by means of a dispensing system from Biodot (Irvine, CA, USA), model AD1500, at 90\% humidity. Irradiation for surface activation and photochemical reaction was carried out at $254 \mathrm{~nm}$ using an UV-Ozone cleaning system (UVOH150 LAB, FHR, Ottendorf-Okrilla, Germany) without oxygen flushing (light intensity achieved $>50 \mathrm{~mW} / \mathrm{cm}^{2}$ ).

Static contact angles ( $\theta$, mean \pm standard deviation of 12 replicates) were measured with a DinoLite camera connected to Dinocapture 2.0 software (AnMo Electronics Corporation, Taiwan), with 3 $\mu \mathrm{L}$ drops of three probe liquids: ultrapure water $(18 \mu \Omega)$, glycerol and diiodomethane. Fluorescence was scanned by means of a home-made Surface Fluorescence Reader (SFR) with excitation filter at 595-620 nm and emission filter at 650-700 nm ranges [10], and attained images were treated with TotalLab TL120 V2008 (Newcastle, UK, http://totallab.com/) software package. 


\section{Surface silanization and characterization}

Surface modification was accomplished by liquid phase reaction in toluene. Prior to silanization, glass surfaces (chips) were rinsed with ethanol, blow dried and activated with UV irradiation for 10 min. Glass surfaces were incubated for two hours with a $1 \%$ silane solution in toluene, rinsed with 2propanol and blow dried. Finally, chips were cured overnight at $160{ }^{\circ} \mathrm{C}$ and stored dry at room temperature until use. For comparison a treatment with toluene only (no silane) was included. When using mixtures of silanes, the total amount of silane was kept to $1 \%$.

Surface roughness was determined by profilometry for the original raw slides. Also, silanized smooth chips were analyzed by X-ray photoelectron spectroscopy (XPS) for atom percentage and C1s peak deconvolution (see details in ESM, S2). Surface hydrophobicity and energy was stated by measuring the static contact angle $(\theta$, mean \pm standard deviation of 12 replicates) with $3 \mu \mathrm{L}$ drops of three probe liquids: ultrapure water $(18 \mu \Omega)$, glycerol and diiodomethane.

\section{Biotin anchoring and binding studies}

For biotin immobilization, multiple drops (50 nL) of TB compound in PBSG were arrayed on the silanized surfaces and irradiated at $254 \mathrm{~nm}$ for $30 \mathrm{~s}$ for thiol-ene reaction. Distance between drops (and within rows) was $1.5 \mathrm{~mm}$ (ESM, Fig. S3). After washing with PBST and water, and blow drying, streptavidin binding was carried out by covering the chips with a Cy5-streptavidin solution ( $20 \mu \mathrm{L}, 25$ $\mathrm{mg} / \mathrm{L}$ in PBST) for $1 \mathrm{~h}$ at room temperature in the dark, followed by another washing and drying step. For the biotin/antibodies interaction studies, after biotin anchoring specific rabbit anti-biotin antibody (20 $\mu \mathrm{L}, 20 \mathrm{mg} / \mathrm{L}$ in PBST) was added and incubated for $1 \mathrm{~h}$ at room temperature, followed by another addition and incubation ( $1 \mathrm{~h}$ in the dark) of Alexa-Fluor labelled goat anti-rabbit secondary antibody $(20 \mu \mathrm{L}, 20 \mathrm{mg} / \mathrm{L}$ in PBST).

The fluorescence of the bound fluorescent labels was displayed as white spots with the SFR and the resulting image was treated to achieve spot intensity, spot diameter and signal-to-noise ratio (SNR, average related to background).

\section{Results and discussion}

\section{Surface silanization and characterization}

In order to maximize the amount of free alcohol -OH groups and achieve a high silanization degree, the activation of chips was performed successfully by irradiating the surface with UV (254 nm) high intensity radiation in the ozone surface cleaner. Indeed, after 10 min radiation, the resulting WCA of glass dropped to $10-15^{\circ}$. 
The wet silanization procedure used was easy and effective. The coating of the surface with silane was checked by XPS, as well as by measuring the hydrophobicity (WCA) of the resulting surface. Indeed, XPS analysis showed that silanization increased the percentage of $C$ atoms at the surface, decreasing the $\mathrm{Si}$ one, and the presence of the $\mathrm{C}=\mathrm{C}$ bonds could be deduced from recorded spectra. Also, when using fluorinated silanes the observed $\mathrm{C}$ percentage was lower but a clear signal for $\mathrm{F}$ atoms was registered. Detailed information can be found in ESM S2.

Wettability of derivatized glass, described by WCA data, is shown in Table 1. Values show that coating glass surfaces with silanes, thus replacing polar -OH groups by nonpolar hydrocarbons, lowers surface hydrophilicity. WCA of surfaces increased between $30^{\circ}$ and $70^{\circ}$ with silanization. As expected, the larger the hydrocarbon chain is, the higher the WCA value and therefore the more hydrophobic the surface is [11]. An exception was $\mathrm{C}_{04}$-BTES silane, which did not react properly with activated glass as suggested by both WCA and XPS data, supposedly due to the ethoxy leaving group of this silane, worse performing that the methoxy one [12]. Anyway, no high hydrophobicity was reached, and the highest WCA values (around $100^{\circ}$ ) were achieved for the two fluorosilanes assayed.

Concerning wettability dependence on surface roughness, using frosted glass, published studies show that the creation of micro/nanostructures on the surface increase hydrophobicity in hydrophobic surfaces, and hydrophilicity in hydrophilic ones [13]. Our results show that roughness enhances hydrophobicity for large silanes (hydrophobic) and hydrophilicity for short ones (hydrophilic), but as frosted glass has microstructures instead of micro/nanostructures, the increase is lower than expected.

The complete characterization of derivatized supports was carried out by calculating the surface tension values for each one, the total value $\left(\gamma_{S}^{\text {Total }}\right)$ and its nonpolar (van der Waals, $\gamma_{S}^{L W}$ ) and polar (acid-base, $\gamma_{S}^{A B}$ ) components. A full description of this study, discussion and conclusions can be found in ESM, S4.

\section{Biotin anchoring and recognition assays}

For studying the influence of surface properties on anchoring and binding assays, the first approach was arraying TB derivative at decreasing concentrations on the different silanized chips, irradiating for thiol-ene link, and further reaction with Cy5-streptavidin conjugate. Fig. 1 shows the Cy5 fluorescence recordings displayed on the SFR images achieved for silanized smooth surfaces. Images obtained with frosted glass are found in ESM S5.1. 


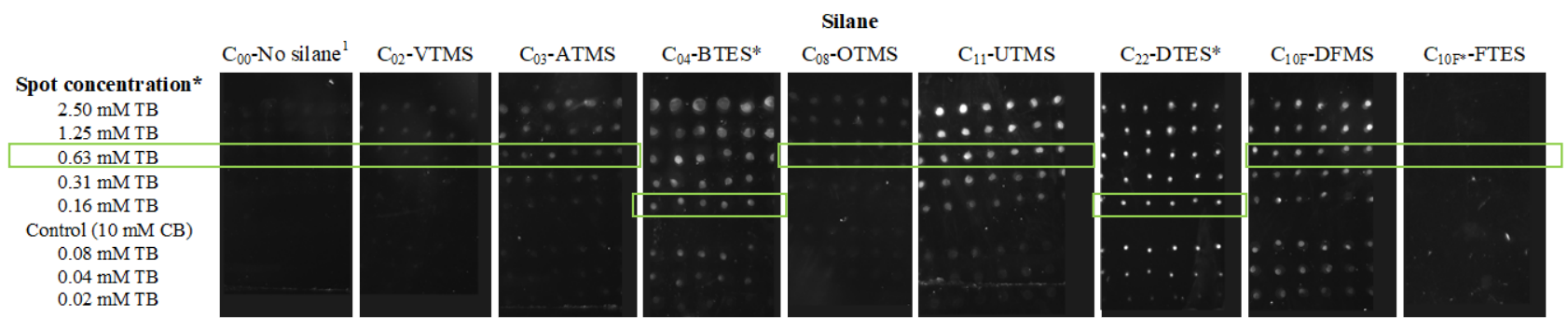

Fig. 1 Cy5-streptavidin spots registered with all the silanized smooth glass surfaces (left: amount of reagent deposited in each droplet). The marked rows correspond to spots achieved with TB at concentration $0.63 \mathrm{mM}$, employed for quantitative data (Table 1). Treatments with * use $10 \mathrm{mM}$ as the highest TB concentration (instead of $2.50 \mathrm{mM}$ ) and decreasing concentrations by a factor of two.

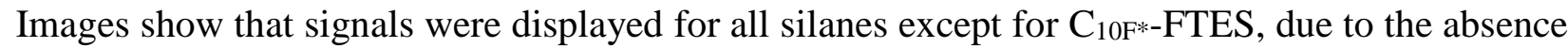
of surface vinyl for the thiol-ene reaction. The minimal naked-eye clearly detectable concentration of TB compound was very low ( $0.08 \mathrm{mM}$ and lower). The image software treatment allowed to quantify the spots, and numerical results are listed in Table 1 for the biotin-streptavidin system on smooth and frosted glass.

The best results obtained regarding high SNR and small spots were achieved with the largest hydrocarbon chain alkenes $\mathrm{C}_{11}$ and $\mathrm{C}_{22}$, and the fluorinated one $\mathrm{C}_{10 \mathrm{~F}}$-DFMS, corresponding to the most hydrophobic surfaces. Anyway, shorter silanes such as allyl C03-ATMS led to acceptable immobilization and binding results. It seems clear that surface mild hydrophobicity is favorable for both biotin anchoring and streptavidin binding. For this, the choice of the silane achieving this wettability improves the results. For frosted glass, spots registered were bigger, the spot size being nearly the same for all silanized surfaces, no matter its hydrophobicity. Intensity values and the background level were also higher, so the SNR values were in general lower. Hence, the employment of rough frosted glass did not lead to any advantage, so the rest of experiments were carried out only with conventional smooth glass chips. 
Table 1 Glass surfaces derivatized with the different silanes. WCA data and Cy5 signals registered with the biotin-streptavidin system.

\begin{tabular}{|c|c|c|c|c|c|c|}
\hline Surface & & WCA $\left(^{\circ}\right)$ & Spot area $^{1}$ & Signal intensity ${ }^{1,2}$ & $\mathrm{SNR}^{1}$ & Lowest $[\mathrm{TB}]^{3}$ \\
\hline \multirow{9}{*}{ 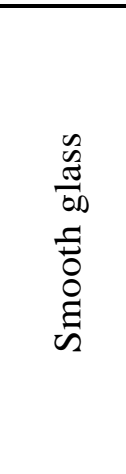 } & $\mathrm{C}_{00}{ }^{4}$ & $18 \pm 6$ & 0.41 & $0.04 \pm 0.05$ & - & - \\
\hline & $\mathrm{C}_{02}$-VTMS & $45 \pm 4$ & 0.17 & $0.89 \pm 0.17$ & 54 & 0.15 \\
\hline & $\mathrm{C}_{03}$-ATMS & $60 \pm 3$ & 0.23 & $2.4 \pm 0.6$ & 112 & 0.04 \\
\hline & $\mathrm{C}_{04}$-BTES & $46 \pm 4$ & 0.25 & $7.4 \pm 1.2$ & 73 & 0.04 \\
\hline & $\mathrm{C}_{08}$-OTMS & $63 \pm 3$ & 0.23 & $2.5 \pm 0.3$ & 51 & 0.04 \\
\hline & $\mathrm{C}_{11}$-UTMS & $84 \pm 3$ & 0.21 & $12.1 \pm 1.5$ & 237 & 0.08 \\
\hline & $\mathrm{C}_{22}$-DTES & $87 \pm 5$ & 0.12 & $6.4 \pm 0.8$ & 787 & 0.08 \\
\hline & $\mathrm{C}_{10 \mathrm{~F}}$-DFMS & $89 \pm 4$ & 0.19 & $8.7 \pm 1.6$ & 1174 & $<0.02$ \\
\hline & $\mathrm{C}_{10 \mathrm{~F}^{*}-\mathrm{FTES}}$ & $104 \pm 3$ & - & - & - & - \\
\hline \multirow{9}{*}{ 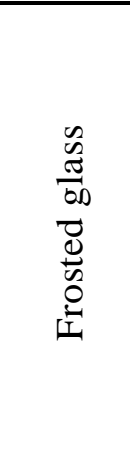 } & $\mathrm{C}_{00}{ }^{4}$ & $34 \pm 4$ & 0.25 & $3.0 \pm 0.9$ & 17 & - \\
\hline & $\mathrm{C}_{02}$-VTMS & $45 \pm 6$ & 0.40 & $8 \pm 2$ & 230 & $<0.02$ \\
\hline & $\mathrm{C}_{03}$-ATMS & $51 \pm 4$ & 0.46 & $1.9 \pm 0.4$ & 57 & $<0.02$ \\
\hline & $\mathrm{C}_{04}$-BTES & $42 \pm 3$ & 0.35 & $10 \pm 2$ & 221 & $<0.02$ \\
\hline & $\mathrm{C}_{08}$-OTMS & $66 \pm 5$ & 0.46 & $3.2 \pm 0.4$ & 196 & $<0.02$ \\
\hline & $\mathrm{C}_{11}$-UTMS & $90 \pm 9$ & 0.33 & $8 \pm 2$ & 240 & $<0.02$ \\
\hline & $\mathrm{C}_{22}$-DTES & $88 \pm 8$ & 0.33 & $12 \pm 2$ & 160 & $<0.02$ \\
\hline & $\mathrm{C}_{10 \mathrm{~F}}-\mathrm{DFMS}$ & $101 \pm 8$ & 0.37 & $14 \pm 3$ & 100 & $<0.02$ \\
\hline & $\mathrm{C}_{10 \mathrm{~F}^{*}-\mathrm{FTES}}$ & $115 \pm 12$ & - & - & - & - \\
\hline \multirow{8}{*}{ 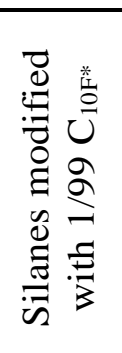 } & $\mathrm{C}_{00}{ }^{4}$ & $109.1 \pm 0.6$ & - & - & - & - \\
\hline & $\mathrm{C}_{02}$-VTMS & $108.0 \pm 0.8$ & 0.08 & $0.21 \pm 0.12$ & 27 & 0.04 \\
\hline & $\mathrm{C}_{03}$-ATMS & $108.7 \pm 1.3$ & 0.15 & $0.9 \pm 0.7$ & 683 & 0.04 \\
\hline & $\mathrm{C}_{04}$-BTES & $107.4 \pm 0.5$ & - & - & - & - \\
\hline & $\mathrm{C}_{08}$-OTMS & $108.4 \pm 0.7$ & 0.10 & $1.3 \pm 0.4$ & 210 & 0.04 \\
\hline & $\mathrm{C}_{11}$-UTMS & $103 \pm 6$ & 0.17 & $2.3 \pm 1.3$ & 88 & 0.31 \\
\hline & $\mathrm{C}_{22}$-DTES & $108.4 \pm 1.9$ & 0.12 & $10 \pm 2$ & 1760 & 0.02 \\
\hline & $\mathrm{C}_{10 \mathrm{~F}}$-DFMS & $108.3 \pm 0.4$ & 0.15 & $2.7 \pm 0.4$ & 269 & 0.02 \\
\hline
\end{tabular}

${ }^{1}$ Data of spot size $\left(\mathrm{mm}^{2}\right)$, signal intensity (pixel $\left.\times 10^{6}\right)$ and SNR have been determined in all cases for spots achieved with droplets containing TB at $0.63 \mathrm{mM}$.

${ }^{2}$ Each cell shows mean \pm S.D. of nine replicates

${ }^{3}$ The lowest [TB] detected (mM) corresponds to the minimal value showing a SNR $>20$. This is higher than the usual 10 value for establishing the Limit of Quantification of a method, however the aim of the assay is not measuring TB compound concentration, but the binding of ligands to anchored TB; the minimal SNR value higher than 20 assures that the fluorescence spot is clearly distinguished over the background.

${ }^{4} \mathrm{C}_{00}$ glass surface not silanized.

\section{Unspecific binding}

The anchoring/binding experiments described above were also designed to study the unspecific binding of both TB compound and streptavidin. First, the fluorescence signals indicated that Cy5 stayed at places where biotin was immobilized, and unspecific binding of streptavidin on surface was low. Also, for $10 \mathrm{mM}$ control CB compound droplets, no spots were observed, indicating that neither biotin molecule nor the spacer arm interacted with any surface, and the covalent anchoring was due solely to the presence of thiol -SH residue in TB reagent.

As counterpart, slight unspecific binding of TB on glass was observed, because white spots, big and with low intensity, are appreciated on non silanized surfaces (Fig. 1) and even without irradiation (ESM S5.2). This is presumably due to a parasite reaction, the thiol-ol binding, described in the 
literature [14]. As well, the large value of the polar component of the surface would allow a polar molecule such as TB to be adsorbed. However, neither TB nor Cy5-streptavidin bound to glass treated with $\mathrm{C}_{10 \mathrm{~F}}$-FTES, a fluorinated silane without alkene function, (Fig. 1, right and Table 1), indicating again that hydrophobicity hinders unspecific binding.

\section{Modification of surface wettability properties: Effect of irradiation time and biotin anchoring}

Surface properties commented above (Table 1 and Fig. 1) can change when UV radiation reaches the surface or when biotin is anchored on it; due in both cases to a chemical modification of the surface. For this, we studied the effect of UV radiation on wettability of silanized chips, as well as wettability of surfaces covered by biotin.

For the first issue, silanized chips were irradiated for different times, and WCA for the resulting surfaces was plotted vs. irradiation time (Fig. 2). It is noted that, in all cases, intense UV radiation affected the surface and lowered the WCA, but the effect was not the same for the different silanes. Long irradiation times ( $5 \mathrm{~min}$ ) were totally deleterious for all surfaces, that become hydrophilic (WCA $<20^{\circ}$ ). However short irradiation times (30 s) did not affect extensively (WCA decrease $<20^{\circ}$ ) to the fluorinated and $\mathrm{C}_{22}$-DTES silanes, the rest being very sensitive (WCA decrease $>40^{\circ}$ ) even at that short time. If the surface integrity, and therefore hydrophobicity, are to be preserved at the non-receptor zones, it is clearly recommendable to use $\mathrm{C}_{10 \mathrm{~F}}$-DFMS and $\mathrm{C}_{22}$-DTES silanes, as well as a photomask.

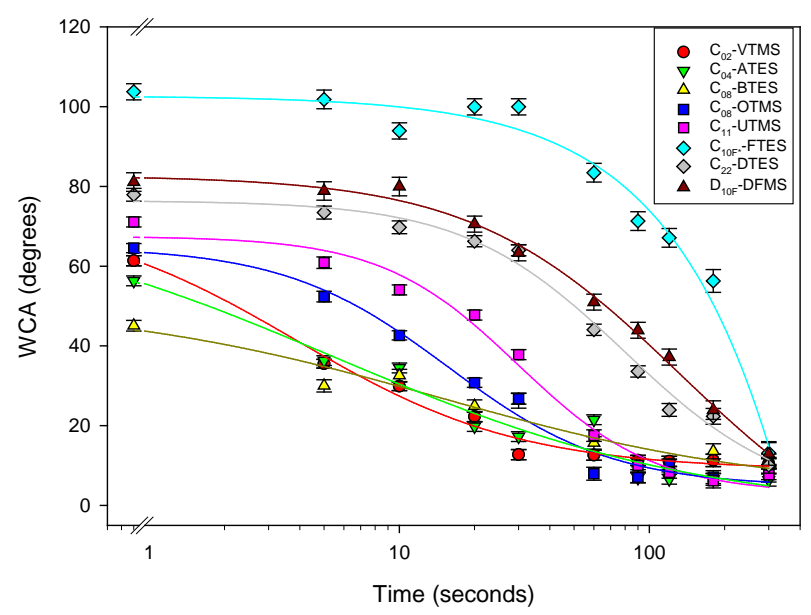

Fig. 2 Evolution of hydrophobicity with UV irradiation time on different silanized surfaces. WCA water contact angle

Surface wettability before and after biotin coating was also determined. In most cases WCA values decreased after TB anchoring, but the reduction was small (less than $10^{\circ}$ except for $\mathrm{C}_{08}$ ) and little significant, indicating that there is not noticeable change in wettability after biotin immobilization. Details are given in ESM, S6. 


\section{Surface derivatization with silane mixtures}

Glass wettability modulation was tried by silanizing smooth glass with mixtures of vinyl $\mathrm{C}_{02}$-VTMS and alkane fluorinated $\mathrm{C}_{10 \mathrm{~F}}$-FTES, in different proportions, and further applying the biotinstreptavidin system as usual. The result was surprising (see spot images at ESM, S5.3) because the same hydrophobicity (WCA $100-110^{\circ}$ ) was achieved for all mixtures, and signal spots were displayed only for very low $(\leq 2.5 \%)$ percentage of $\mathrm{C}_{10 \mathrm{~F}}$-FTES, indicating that the fluorinated silane determines the wettability, and that fluorine chains hinder the streptavidin binding.

Alkene-fluorine chips were later prepared with mixtures of $\mathrm{C}_{10 \mathrm{~F}^{*}-\mathrm{FTES}}$ and all the vinyl silanes at ratio F silane/vinyl silane 1/99 (v/v). Surfaces were assayed for TB anchoring and further streptavidinCy5 recognition (Table 1 bottom and spot images at ESM S5.4). No spots appeared on the control chip, so no unspecific TB binding to the treated surface happened, this result being highly positive, because it means that the use of fluorosilanes is a way to control the primary anchoring reaction and avoid non-desired interactions. Absence of spots on $\mathrm{C}_{04}$ surface indicated the poor behavior of that silane, previously discussed. On the other hand, WCA was $100-110^{\circ}$ for all silanes employed, so addition of $1 \% \mathrm{C}_{10 \mathrm{~F} *}$ produces mild hydrophobic surfaces, their wettability being thus totally decided by this $1 \%$ fluorosilane.

Regarding signals, small sized and low intensity spots were obtained for short silanes ( $\mathrm{C}_{02}$-VTMS, $\mathrm{C}_{03}$-ATMS), while for large silanes (C22-DTES, $\mathrm{C}_{10 \mathrm{~F}}$-DFMS) acceptable intensity spots were displayed. As well, high SNR values were obtained. It can be deduced that the addition of $1 \% \mathrm{C}_{10 \mathrm{~F}^{*-}}$ FTES, completely hinders the unspecific binding of both biotin and streptavidin, but the streptavidin recognition is favored, because the lowest TB concentrations anchored were recognized by streptavidin and, in general, SNR improved.

These results are schematically shown in Fig. 3. When glass is coated only with a short silane (e.g. $\mathrm{C}_{03}$-ATMS) and biotin stands out over the surface due to the TB reagent spacer arm (a), surface is hydrophilic and streptavidin binds both biotin and unspecifically on the surface. If $\mathrm{C}_{10 \mathrm{~F} * \mathrm{FTES}}$ silane is present in a percentage over $2.5 \%$ (b) it controls wettability and totally hinders binding. But if $\mathrm{C}_{10 \mathrm{~F}^{*-}}$ FTES silane is in a 1\% percentage (c), sterptavidin only binds to biotin and not to the surface, thus improving SNR. When coating with a larger silane such as $\mathrm{C}_{08}$ or $\mathrm{C}_{11}$ with $1 \% \mathrm{C}_{10^{*}}(\mathrm{~d})$, the effect of $\mathrm{C}_{10 \mathrm{~F}}$ silane is not so determinant, because hydrophobicity and recognition ability are similar. 

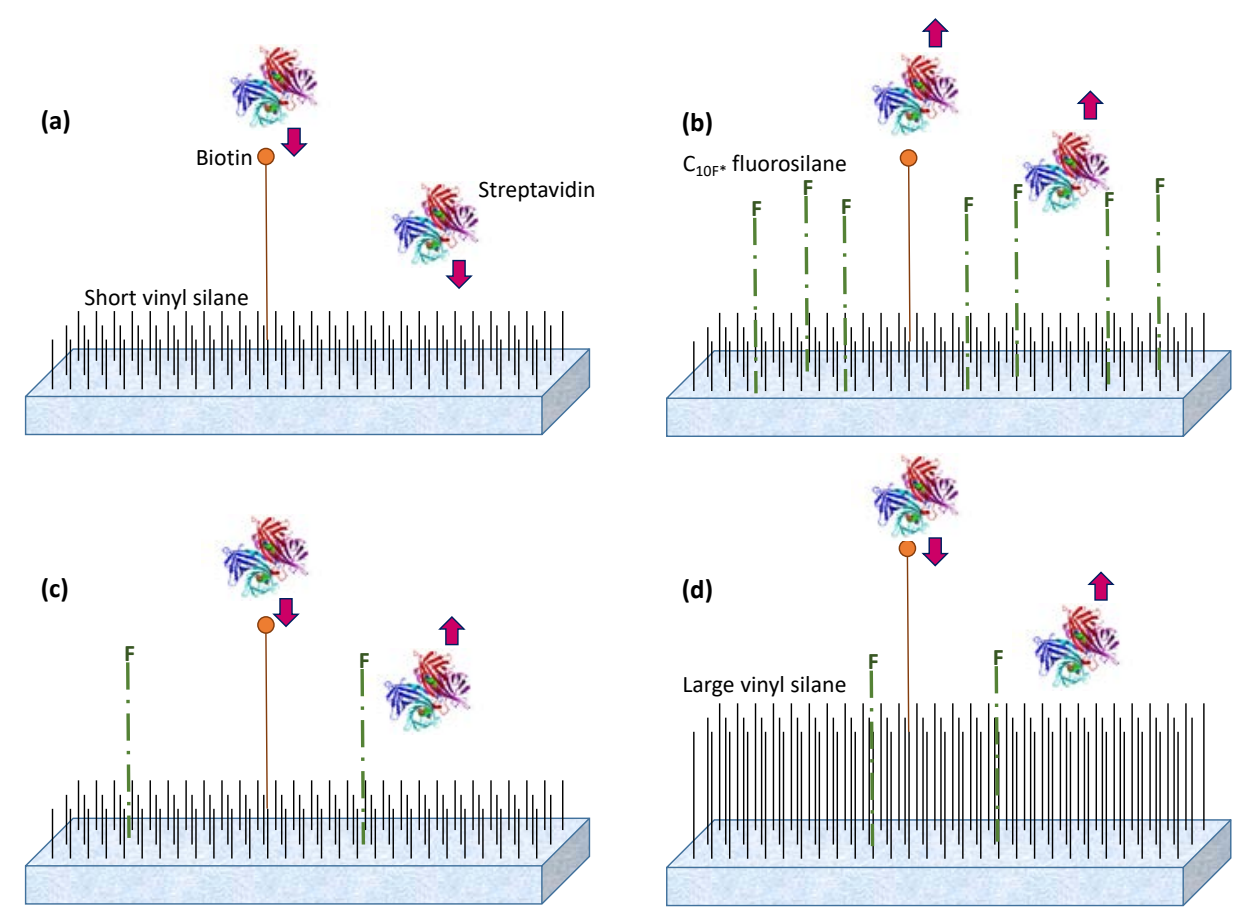

Fig. 3 Topography of silanized surfaces and recognition assay. (a) Short silane alone. (b) Short silane modified with $>2.5 \% \mathrm{C}_{10 \mathrm{~F} *-F T E S}$. (c) Short silane modified with $\leq 2.5 \% \mathrm{C}_{10 \mathrm{~F} *-\mathrm{FTES}}$. (d) Large silane modified with $\leq$ $2.5 \% \mathrm{C}_{10 \mathrm{~F} *-\mathrm{FTES}}$.

\section{Study of the biotin/antibody/secondary antibody system}

In order to state the real value of the study performed with the biotin-streptavidin system, a similar procedure was applied in an indirect immunoassay, where arrayed immobilized biotin (hapten) was reacted with specific anti-biotin antibody, and a fluorescently labelled secondary antibody was further added. This system was assayed for all vinyl silanes on smooth glass except $\mathrm{C}_{04}$-BTES, alone and modified with $1 \% \mathrm{C}_{10 \mathrm{~F}}$-FTES. Fluorescence spots achieved can be seen in ESM S7, and numerical data achieved are shown in Table 2.

Results obtained show some differences if compared to streptavidin binding model, although the general trend is the same. Absolute intensity values are lower, as expected since the bioreagents have different affinity and behavior, and the spots are in general smaller, all this being traduced in lower SNR values. However, signal and SNR values increase with hydrophobicity, and results are maximal for $\mathrm{C}_{22}$-DFMS treatment, similarly to biotin-streptavidin interaction. A great difference between the two interactions appears for the fluorosilane $\mathrm{C}_{10 \mathrm{~F}}$-DFMS, with highest SNR for streptavidin and lowest one for antibody, possibly due to the rejection of antibodies (much bigger than streptavidin) by fluorinated surface. This result indicates that the parallelism is not absolute and surface conditions must be checked.

Regarding non-specific binding, the background fluorescence from secondary antibody is higher than that obtained with Cy5-streptavidin, because the antibody is a protein bigger than streptavidin, with more polar aminoacid residues on the surface, so the conjugate is more prone to adsorb on the surface, and the SNR is lower. However, general background decreases when hydrophobicity increases, rising SNR in all cases. 
Table 2 Glass surfaces derivatized with the different silanes. Alexa-Fluor 647 signals registered with the biotin/antibody/secondary antibody system

\begin{tabular}{|c|c|c|c|c|c|c|c|c|}
\hline \multirow[b]{2}{*}{ Surface } & \multicolumn{4}{|c|}{ Pure silane } & \multicolumn{4}{|c|}{ Silane with $1 / 99 \mathrm{C}_{10 \mathrm{~F} *}$} \\
\hline & $\begin{array}{l}\text { Spot } \\
\text { area }^{1}\end{array}$ & $\begin{array}{l}\text { Signal } \\
\text { intensity }\end{array}$ & $\mathrm{SNR}^{1}$ & Lowest $[\mathrm{TB}]^{3}$ & $\begin{array}{l}\text { Spot } \\
\text { area }^{1}\end{array}$ & $\begin{array}{l}\text { Signal } \\
\text { intensity }\end{array}$ & $\mathrm{SNR}^{1}$ & Lowest $[\mathrm{TB}]^{3}$ \\
\hline $\mathrm{C}_{00}{ }^{4}$ & - & - & - & - & -- & - & - & - \\
\hline $\mathrm{C}_{02}$-VTMS & 0.06 & $1.5 \pm 1.0$ & 22 & 0.62 & 0.11 & $4.5 \pm 0.9$ & 2920 & $<0.02$ \\
\hline $\mathrm{C}_{03}$-ATMS & 0.09 & $3.0 \pm 1.7$ & 19 & 0.62 & 0.09 & $0.8 \pm 0.6$ & 21 & 0.62 \\
\hline $\mathrm{C}_{08}$-OTMS & 0.13 & $3.5 \pm 1.4$ & 23 & 0.15 & 0.11 & $2.1 \pm 0.7$ & 91 & $<0.02$ \\
\hline $\mathrm{C}_{11}$-UTMS & 0.19 & $4.7 \pm 1.7$ & 34 & 0.08 & 0.09 & $3.5 \pm 0.8$ & 676 & $<0.02$ \\
\hline $\mathrm{C}_{22}$-DTES & 0.06 & $6.1 \pm 3.3$ & 40 & 0.08 & 0.54 & $4.4 \pm 1.8$ & 169 & 0.04 \\
\hline $\mathrm{C}_{10 \mathrm{~F}}$-DFMS & 0.05 & $1.3 \pm 1.0$ & 21 & 0.62 & 0.09 & $0.9 \pm 0.4$ & 38 & 0.04 \\
\hline
\end{tabular}

${ }^{1}$ Data of spot size $\left(\mathrm{mm}^{2}\right)$, signal intensity (pixel $\left.\times 10^{6}\right)$ and SNR have been determined in all cases for spots achieved with droplets containing TB at $0.63 \mathrm{mM}$.

${ }^{2}$ Each cell shows mean \pm S.D. of nine replicates

${ }^{3}$ The lowest [TB] detected $(\mathrm{mM})$ corresponds to the minimal value showing a SNR $>20$. This is higher than the usual 10 value for establishing the Limit of Quantification of a method, however the aim of the assay is not measuring TB compound concentration, but the binding of ligands to anchored TB; the minimal SNR value higher than 20 assures that the fluorescence spot is clearly distinguished over the background.

${ }^{4} \mathrm{C}_{00}$ glass surface not silanized.

When modifying silane with fluorine, the behavior is similar to that observed for streptavidin system: intensity of spots becomes lower, but non-specific binding is totally removed, so the final result is an improvement of SNR, very high in some cases such as $\mathrm{C}_{02}$-VTMS. This is also traduced in lower TB concentration giving rise to detectable spots.

\section{Conclusions}

Results demonstrate that the proper choice of the derivatizing reagents and surface composition determines the quality of data obtained in binding assays. Glass silanized with short vinyl silanes, results to be mildly hydrophilic at the receptor zone, and more hydrophilic on outer surface, while for large and fluorinated silanes surface is nearly hydrophobic in all regions.

Mild hydrophobic glass surfaces (WCA around 90-100 ${ }^{\circ}$ ) achieved with $\mathrm{C}_{10 \mathrm{~F}}$-DFMS and $\mathrm{C}_{22}$-DTES are by far better than those derivatized with short silanes (C $\mathrm{C}_{02}$-VTMS, $\mathrm{C}_{04}$-BTES). In this work it is confirmed that some hydrophobicity is therefore adequate for avoiding unspecific binding of the protein ligand, and thus preventing background signals and achieving maximum SNR values. Regarding surface roughness, standard microstructured frosted glass does not lead to better results in terms of wettability and binding assays, if compared to normal smooth glass. 
The silanization employing vinyl silanes modified with $1 \%$ of fluorinated $\mathrm{C}_{10 \mathrm{~F} \text {-FTES leads to the }}$ best glass surfaces for the studied bioassay, in terms of optimal surface wettability (mild hydrophobicity), complete absence of unspecific binding of receptor and analyte to the surface, small spots and better SNR.

The gathered information can be applied to other biosensing surfaces derivatizable for applying organosilanes, and also to other protein-protein and hapten-protein biointeractions.

Acknowledgements We thank Patricia Concepción and Dolores Soriano (Instituto de Tecnología Química, Universitat Politècnica de València-Consejo Superior de Investigaciones Científicas) for helping with XPS analysis. Financial support from the Generalitat Valenciana (GVA-PROMETEO/2014/040), FEDER and the Spanish Ministry of Economy and Competitiveness (CTQ2013-45875-R and CTQ2016-75749-R) is acknowledged.

\section{Compliance with ethical Standards}

Conflict of interest All authors declare that they have no competing interests.

Ethical Approval This article does not contain any studies with human participants or animals performed by any of the authors.

\section{References}

1. González-Martínez MA, Puchades R, Maquieira A. Optical immunosensors for environmental monitoring: How far have we come? Anal Bioanal Chem. 2007;387:205-218.

2. Kontogeorgis GM, Kiil S. Intermolecular and interparticle forces. in: Kontogeorgis GM, Kiil S, editors. Introduction to Applied Colloid and Surface Chemistry. Chichester: John Wiley \& Sons; 2016. pp. 1133.

3. Ronkainen NJ, Halsall HB, Heineman WR. Electrochemical immunoassays and immunosensors. in: van Emon JM, editor. Immunoassay and Other Bioanalytical Methods. Boca Raton, FL: CRC Press, Taylor \& Francis Group; 2007. Chapter 16.

4. González-Lucas D, Bañuls M-J, Puchades R, Maquieira A. Versatile and easy fabrication of advanced surfaces for high performance DNA microarrays. Adv Mater Interfaces. 2016;1500850.

5. Yin L, Lu M, Wielunski L, Song W, Tan J, Lu Y, Jiang W. Fabrication and characterization of compact silicon oxynitride waveguides on silicon chips. J Opt. 2012;14:085501. 
6. Zhang F, Sautter K, Larssen AM, Findley DA, Davis RC, Samha H, Linford MR. Chemical vapor deposition of three aminosilanes on silicon dioxide: surface characterization, stability, effects of silane concentration, and cyanine dye adsorption. Langmuir. 2010;26:14648-54.

7. Escorihuela J, Bañuls M-J, Puchades R, Maquieira A. DNA microarrays on silicon surfaces through thiol-ene chemistry. Chem Commun. 2012;48:2116-8.

8. Kontogeorgis GM, Kiil S. Wetting and adhesion. in: Kontogeorgis GM, Kiil S, editors. Introduction to Applied Colloid and Surface Chemistry. Chichester: John Wiley \& Sons; 2016. pp. 121-60.

9. Weinrich D, Köhn M, Jonkheijm P, Westerlind U, Dehmelt L, Engelkamp H, et al. Preparation of biomolecule microstructures and microarrays by thiol-ene .photoimmobilization. ChemBioChem 2010;11:235-47.

10. Mira D, Llorente R, Morais S, Puchades R, Maquieira A, Marti J. High-throughput screening of surfaceenhanced fluorescence on industrial standard digital recording media. in Carrano JC, Zukauskas A, editors. Optically Based Biological and Chemical Sensing for Defence, Proceedings SPIE Vol. 5617. SPIE, Bellingham, WA, 2004, 0277-786X/04/\$15 doi: 10.1117/12.578301.

11. Wasserman SR, Tao YT, Whitesides GM. Structure and reactivity of alkylsiloxane monolayers formed by reaction of alkyltrichlorosilanes on silicon substrates. Langmuir 1989;5:1074-87.

12. Munsch J-N, Dentzer J, Claus W, Bassel H. Structural characterization of silanes modified silica by temperature programmed desorption coupled to mass-spectra (TPD-MS) technique. J Colloid Sci. Biotech. 2014;3:30-7.

13. Celia E, Darmanin T, de Givenchy ET, Amigoni S, Guittard F. Recent advances in designing superhydrophobic surfaces. J Colloid Interface Sci. 2013;402:1-18.

14. Li L, Li J, Du X, Welle A, Grunze M, Trapp O, Levkin PA. Direct UV-induced functionalization of surface hydroxy groups by thiol-ol chemistry. Angew Chem Int Ed. 2014;53:3835-39. 